\title{
Distance Measurement and Energy Conservation Using Arduino Nano and Ultrasonic Sensor
}

\author{
Adekunle Adebola Olayinka ${ }^{1}$, Adekunle Adewale Oluwadamilare ${ }^{2}$, Ayo Femi Emmanuel ${ }^{3}$ \\ ${ }^{1}$ Computer Science Department, School of Science, Adeyemi College of Education, Ondo, Nigeria \\ ${ }^{2}$ Electronic and Electrical Engineering Department, Faculty of Technology, Obafemi Awolowo University, Ile, Ife, Nigeria \\ ${ }^{3}$ Physical and Computer Science Department, College of Natural and Applied Science, McPherson University Seriki Sotayo, Nigeria \\ Email address: \\ gaacademics@gmail.com (A. A. Olayinka), adewaleadekunle439@gmail.com (A. A. Oluwadamilare), \\ emmini8168@gmail.com (A. F. Emmanuel)

\section{To cite this article:} \\ Adekunle Adebola Olayinka, Adekunle Adewale Oluwadamilare, Ayo Femi Emmanuel. Distance Measurement and Energy Conservation \\ Using Arduino Nano and Ultrasonic Sensor. American Journal of Electrical and Computer Engineering. Vol. 5, No. 2, 2021 , pp. $40-44$. \\ doi: 10.11648/j.ajece.20210502.11
}

Received: November 23, 2020; Accepted: July 5, 2021; Published: July 13, 2021

\begin{abstract}
This research work is designed as the distance measurement with energy conservation system using Ultrasonic sensor and Arduino NANO. Ultrasonic sensor emits high frequency sound waves, which reflects from target surfaces. This work utilized these sound waves through Ultrasonic sensor HC-SR04 to determine the change in distance and to apply it to detect the presence of an obstacle (person in this case), which in turns triggers a light bulb ON. Sonar waves are projected back to the receiving end of the sensor after which electrical pulses emitted from the sensor are sent into the Arduino NANO board, electrical signals are then sent to the LEDs and lighting system (bulbs). This work shows the importance of distance measurement to an automatic, hands-free environment control, efficient use of energy sources, and conservation of energy. The experimental results have shown minimal errors $(<3 \mathrm{~cm})$ for distances between $10 \mathrm{~cm}$ and $100 \mathrm{~cm}$ as the research deployment is most suitable within these distances. The results also describe the corresponding responses of the control system to the different physical conditions likely to be present. The above processes focus on electrical energy conservation as the light bulb comes ON when a person approaches the sensing system and goes OFF when the person leaves.
\end{abstract}

Keywords: Arduino NANO, Distance, Energy, HC-SR04

\section{Introduction}

Measurement in today's world is the pivot in control systems as is vital in controlling devices to one's taste. Measurement spans Physical/environmental measurands including temperature, distance, light, water/moisture content, blood pulses, height, weight, volume and so on. Distance measurement has led to significant contributions in control systems. Distance measurement sensors are used to control or indicate the position of objects, people and obstacles. A useful application of distance measurement with Ultrasonic sensors is automatic lighting for electrical energy conservation. Distance measurement sensors can determine the dimensions of objects such as height, width and diameter, using one or more sensors. Ultrasonic sensor provides a reliable approach to distance measurement. The sensor is perfect for distance measurements between moving or stationary objects. Ultrasonic Sensors measure the distance of the objects in air through non-contact technique. They measure distance without damage and are easy to use. Ultrasonic sensors are widely used for distance measurement purposes. They are classified in the group of transducers which can work at a considerable amount of range [5].

They offer low cost and a precision of less than $1 \mathrm{~cm}$ in distance measurements of up to $450 \mathrm{~cm}$. However, the most popular method used in these measurements is based on the time of flight (ToF) measurement. This ToF is the time elapsed between the emission and subsequent arrival after reflection of an Ultrasonic pulse train travelling at the speed of sound [1]. This causes large response times for a single measurement. Light bulbs in walkways are usually ON while no one passes by which should be avoided. A useful way to prevent energy wastage from the light bulbs is using the 
ultrasonic sensor to detect change in distance as passer by approaches or leaves the propagation distance established by the sensor interfaced with a processor. Arduino NANO board is a flexible, compatible, user-friendly board that runs on an ATMEGA 328P processor. Owing to the constant loss of energy where not needed, it deems necessary to develop an optimal approach to conserve energy effectively where cost of paying for such energy is expensive.

\section{Implementation}

This section will be sub-divided into two parts; the hardware description and the software description.

Hardware implementation

The hardware implementation comprised of the components of the system circuit, from the input stage to the output stage. The list of components and their part numbers are stated in the table below:

Table 1. List of components.

\begin{tabular}{|c|c|c|c|}
\hline No. & Name & $\begin{array}{l}\text { Part Number / } \\
\text { Spec }\end{array}$ & Quantity \\
\hline 1. & Ultrasonic sensor & HC-SR04 & 1 \\
\hline 2. & Arduino & NANO & 1 \\
\hline 3. & $\begin{array}{l}\text { RGB LED or Red, Green and } \\
\text { Yellow LED }\end{array}$ & --- & $1 / 3$ \\
\hline 4. & Light bulb & $12 \mathrm{~V} / 220 \mathrm{~V}$ & 1 \\
\hline 5. & Resistors & $220 \mathrm{ohms}$ & 3 \\
\hline
\end{tabular}

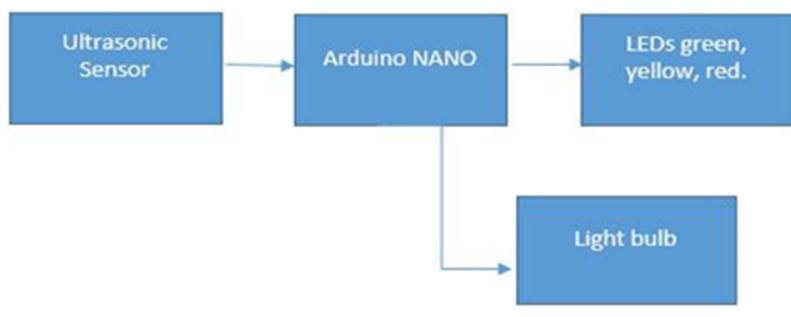

Figure 1. Block diagram of the hardware implementation.

The Arduino NANO is the brain of the entire system. It accepts the input, i.e. from the Ultrasonic sensor (HC-SR04) and processes the data according to the requirements and provides the necessary action. The $\mathrm{C}$-based simple program code for the Arduino is referred to as a sketch [2]. The output block comprises of the LEDs, Light bulbs. Depending upon the output received from the Arduino, the different units of the output block will be triggered.

The table below describes the specifications of the Arduino NANO board;

Table 2. Specifications of the Arduino NANO board.

\begin{tabular}{ll}
\hline Processor & ATmega328P \\
\hline Operating Voltage & $5 \mathrm{~V}$ \\
Input Voltage & $7 \mathrm{~V}-12 \mathrm{~V}$ \\
CPU speed & $16 \mathrm{MHz}$ \\
Analog In/Out & $8 / 0$ \\
Digital IO/PWM & $14 / 6$ \\
EEPROM/SRAM & $1 / 2$ \\
Flash & 32 \\
USART & 1 \\
\hline
\end{tabular}

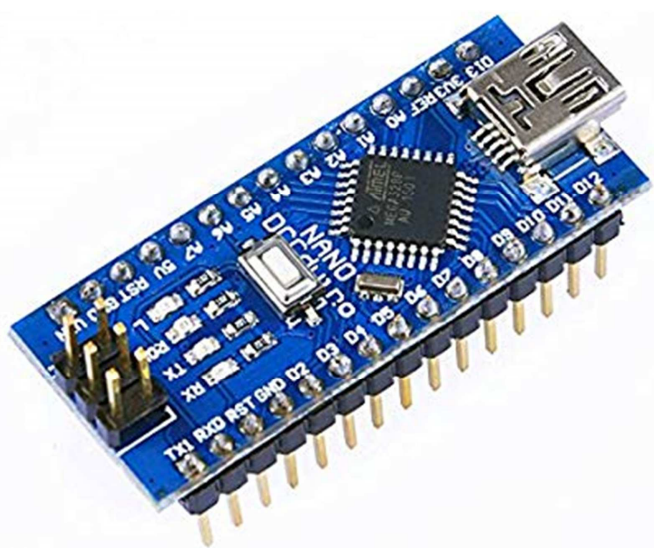

Figure 2. The Arduino NANO board.

This application is based on the reflection of sound waves emitted by the ultrasonic sensor module HC-SR04. The HCSR04 uses SONAR to determine the distance of an object just like the bats do [3]. The sensor emits an ultrasound at 40 $\mathrm{KHz}$ which travels through the air and if there is an object or obstacle on its path, it will bounce back to the module. Considering the travel time and the speed of the sound, you can calculate the distance. Below is the schematic for the hardware setup;

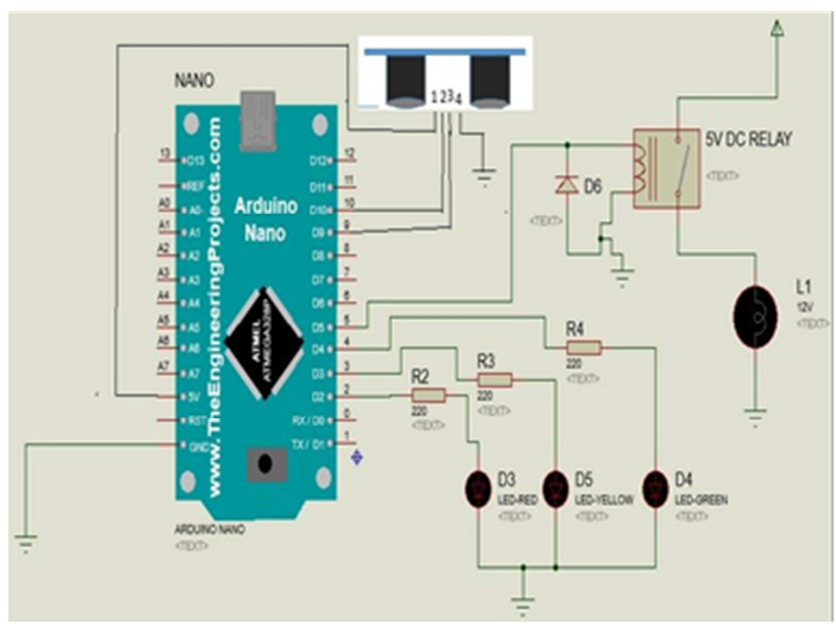

Figure 3. Circuit diagram of the hardware implementation.

Here, the ultrasonic sensor is connected to the NANO board's digital pins D9 and D10. The Trigger pin (Pin 2) of the ultrasonic sensor is connected to D10, the echo pin (Pin 3) is connected to D9.

The VCC pin (Pin 1) is connected to the $5 \mathrm{~V}$ pin of the NANO board as the Ultrasonic sensor is powered with $5 \mathrm{~V}$. The ground pin (Pin 4) is connected to the ground of the NANO board. The trigger pin of the ultrasonic sensor is used to send pulses out and as it strikes any object, it bounces back to the sensor, and the echo pin sends the pulses into the microcontroller. The rate at which pulses are sent into the microcontroller is used to calculate the distance between the sensor and the object or obstacle. The distance or change in distance in this case is the measurement parameter used in the control system. 


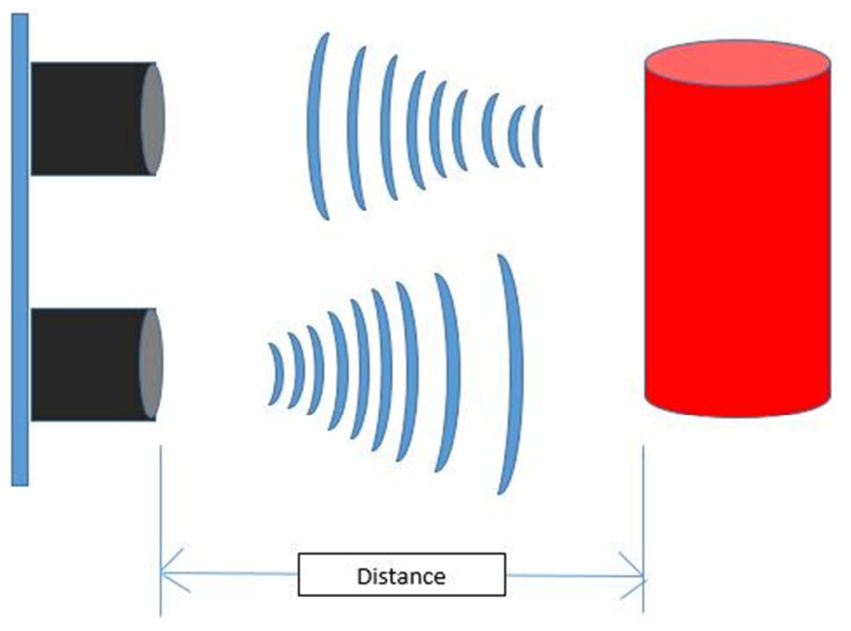

Figure 4. Operation of the Ultrasonic sensor.

Table 3. Pinout of the Ultrasonic sensor.

\begin{tabular}{ll}
\hline Pin 1 & VCC \\
Pin 2 & Trigger \\
Pin 3 & Echo \\
Pin 4 & Ground \\
\hline
\end{tabular}

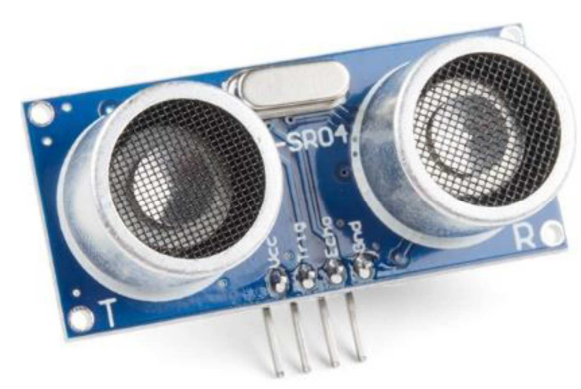

Figure 5. The Ultrasonic sensor.

The red LED is connected to Pin 2 of the NANO board, the yellow LED is connected to PIN 3 of the board, the green LED is connected to Pin 4 of the NANO board. Resistors are connected in series with the LEDs to limit the current flow into LEDs. The three LED pins are OUTPUT pins, they display the change in distance detected by the sensor as the detected objects approaches the sensor or moves away from the sensor. The Light bulb is a $220 \mathrm{~V}$ bulb only that it will not be controlled directly by the microcontroller. This is because the maximum voltage output from the microcontroller is $5 \mathrm{~V}$ and it will not be capable of meeting the current requirements of the bulbs. Therefore, an external power supply powers the light bulb. However, a $5 \mathrm{~V}$ relay is most suitable for this purpose. It switches the power supply to the light bulb with respect to the voltage output from the microcontroller. The diode connected to the relay is a protective diode called the flyback diode. Whenever voltage is supplied to a coil and current flows, it generates voltage spikes that returns to the power supply. The diode creates low resistance path that prevents the voltage spikes from returning to the power supply. These voltage spikes could create disorder in the control circuit, destroy the power supply and in subsequent cases, lead to misconception of problems in the circuit design. The software implementation

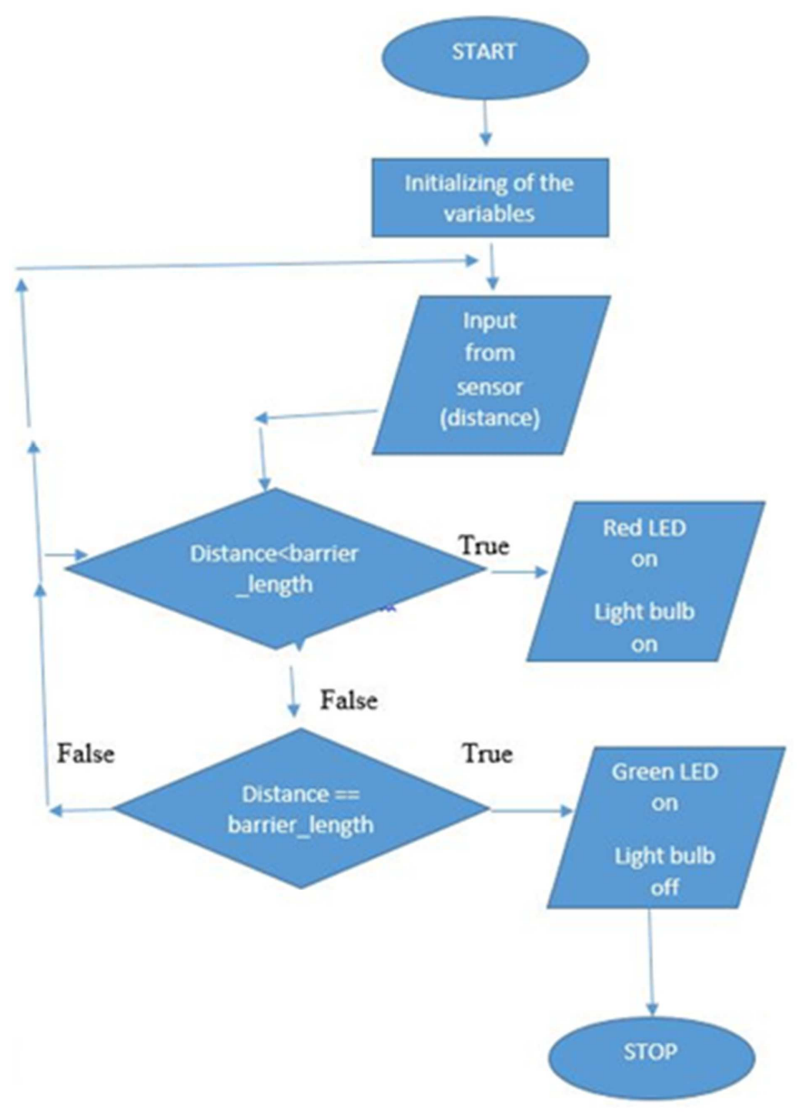

Figure 6. Control Software implementation Flow Chart.

The variable initialization includes declaration of the barrier length which is gotten by placing the ultrasonic sensor at a constant distance position. This implies there is a threshold distance established by the position of the sensor. As an obstacle is detected, there will only be a decrease in the established distance which is therefore less than the barrier length. The Red LED comes on when an obstacle is detected, the light bulb also comes on and remains on for a specified delay, after which it goes off automatically. Just before the delay time ends, the Yellow LED comes on, and goes OFF after the delay time.

(The yellow LED response wasn't included in the Flow Chart because it didn't involve a response targeted at controlling anything).

For any instance in which there is no detected obstacle i.e. there is no decrease in barrier length, the Green LED remains while the light bulb remains off until there is an obstacle detected.

\section{Results and Analysis}

The section contains results and readings obtained during the experiment. 
Table 4. Experimental Results of Ultrasonic sensor for Various Distances.

\begin{tabular}{|c|c|c|c|c|c|}
\hline \multirow{2}{*}{ Distance (cm) } & \multicolumn{5}{|c|}{ Ultrasonic Sensor readings $(\mathrm{cm})$} \\
\hline & Experiment 1 & Experiment 2 & Experiment 3 & Average & Absolute Error \\
\hline 10 & 10 & 9.8 & 10 & 9.93 & 0.07 \\
\hline 20 & 21 & 20 & 23 & 21.33 & 1.33 \\
\hline 30 & 31 & 32 & 30 & 31.00 & 1.00 \\
\hline 40 & 40 & 40 & 41 & 40.33 & 0.33 \\
\hline 50 & 52 & 52 & 51 & 51.67 & 1.67 \\
\hline 60 & 63 & 61 & 60 & 61.33 & 1.33 \\
\hline 70 & 72 & 73 & 69 & 71.67 & 1.67 \\
\hline 80 & 85 & 80 & 82 & 82.33 & 2.33 \\
\hline 90 & 90 & 96 & 92 & 92.67 & 2.67 \\
\hline 100 & 102 & 102 & 104 & 102.67 & 2.67 \\
\hline
\end{tabular}

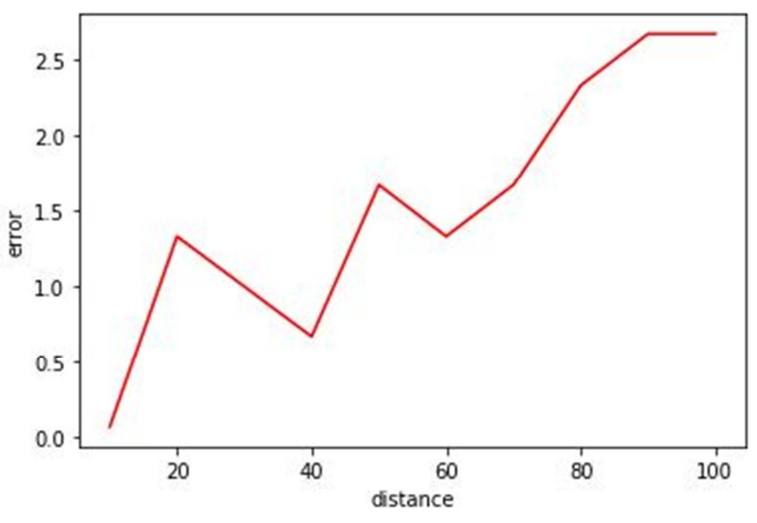

Figure 7. Error vs Distance Plot of the Ultrasonic sensor Readings.

Table 5. LED and Light Bulb Responses.

\begin{tabular}{llll}
\hline No. & Ultrasonic Reading & LED & Response \\
\hline 1. & Distance $<$ barrier_length & Red & Light bulb ON \\
2. & Distance=barrier_length & Green & Light bulb OFF \\
\hline
\end{tabular}

\section{Conclusions}

This study is aimed to obtain the distance measurement of the ultrasonic sensor with respect to the presence of obstacles or obstructions and in addition to this, to control a set of devices due to the distance measured by the sensor.

Moreover, the study seek to find the significance and impact of the materials used in correlation with the ability of the ultrasonic sensor to measure distance precisely. Furthermore, this study could benefit anyone interested in conserving electrical energy without the use of human presence by applying smart algorithms for obstacle detection, precise detection of an object.

In this study, a tool to detect motion of objects, light by LEDs was assembled. The circuit was successfully connected and the program was sent to the Arduino NANO microcontroller board to run the circuit. The ultrasonic sensor was able to send the ultrasonic sound waves to the approaching object. LEDs were also set to produce light signals in a particular set of distances starting with Green LED then the Red LED. On the outcome, LEDs produced light as they were expected. The Light bulb was also expected to come on during the variation of distances as the object approaches the ultrasonic sensor. This tool can be custom made to fit various applications such as being fitted in houses to aid when little or no man power is present, could tell a house owner if an obstacle intersects the barrier length whenever the light bulb comes on etc. The technique can also be implemented with the GSM for proper remote monitoring. The technique can also be recommended to be used in the tunnels such as train or vehicle tunnels to give signal in case there are movements. The ultrasonic sensors can measure distances through moderate amounts of dust, smoke and humidity as seen in [15]. Other applications of the ultrasonic sensor include water level detection [4], guided rocket objective finding [5], lock detection on RADAR systems [6], volume level monitoring [8], obstacle detection [9], selfdriving cars [10, 11], human presence detection [12], robotic sensing [13, 17], Rain water harvesting [14], collision protection [7].

\section{References}

[1] Christofer N. Y., Mathew S. A (2017). "Analysis of Obstacle Detection Using Ultrasonic Sensor". International Research Journal of Engineering and Technology (IRJET), 4 (1), Pp. $1015-1019$.

[2] Latha N. A., Murthy B. R, Kumar K. B (2016) "Distance Sensing with Ultrasonic Sensor and Arduino" International Journal of Advanced Research, Ideas and Innovations in Technology Volume 2, Issue 5, pp. 1-5.

[3] Mutava M., Paul K. (2020) "Arduino Uno, Ultrasonic Sensor HC-SR04 Motion Detector with Display of Distance in the LCD" International Journal of Engineering Research \& Technology, Vol. 9 Issue 05, pp 936-942.

[4] Bello M. I., Gana S. M., M. I. and Faruk M. I., Umar M. J. (2018). "AUTONOMOUS ULTRASONIC BASED WATER LEVEL DETECTION AND CONTROL SYSTEM" Nigerian Journal of Technology, Volume 37, No. 2, pp. 508-513.

[5] Khudaija T. M., Hamza I. (2017): "DESIGNING OF LOW COST ARDUINO BASED ULTRASONIC SONAR SYSTEM" VFAST Transactions on Software Engineering Volume 12, No 3, pp. 57-65.

[6] Baskoro F, Reynaldo B R (2016). "Detection of Lock on Radar System Based on Ultrasonic US 100 Sensor And Arduino Uno R3 With Image Processing GUI", IOP Conference Series: Materials Science and Engineering, pp 0105 . 
[7] Neeraja S., Sarita C. H., Shrikant M., Basant S. S., Bhavishya J., Garima S (2017). "Distance Measurement using Ultrasonic Sensor and Arduino" International Journal of Engineering Science and Computing, Volume 07, Issue No. 3, pp 59915992.

[8] Husni M., Siahaan D. O, Ciptaningtyas H. T, Studiawan H and Aliarham Y. P (2016). "Liquid volume monitoring based on ultrasonic sensor and Arduino microcontroller" International Conference on Innovation in Engineering and Vocational Education. IOP Science, iopscience. iop. org, pp 01-10.

[9] Vidhya D. S., D’Silva C. J., Rebelo D. P., Fernandes L. W. (2016). "Obstacle Detection using Ultrasonic Sensors" International Journal for Innovative Research in Science \& Technology Volume 2, Issue 11. pp 316-320.

[10] Parveen K., Animesh D., Manash P. B., Smriti D. (2019). "Smart Vehicle System using Arduino" ADBU Journal of Electrical and Electronics Engineering Volume 3, Issue 1. pp. 20-25.

[11] Roni S., Adhi K., Nina S. A., Mitra D., Sparisoma V. (2017). "An Ultrasonic Sensor System for Vehicle Detection Application" Roni Stiawan et al J. Phys.: Conf. Ser. 1204 012017, pp 01-06.

[12] T. V Groeningen, H. Driessen, J. Sohl, R. Voute (2018). “An ultrasonic sensor for human presence detection to assist rescue work in large buildings" ISPRS Annals of the Photogrammetry, Remote Sensing and Spatial Information Sciences, Volume IV-4/W7 pp 135-140.

[13] Hassan M., Reza A., Mahmoud O., Seyed S. M. and Noboru N. (2011) "Determination of Ultrasonic Sensor Ability for Use as Guidance Sensors of Mobile Robots" Sensors and Materials, Vol. 24, No. 3 (2012) pp 115-126.

[14] Kalyan D., V S P Chandrika K., Chinna R. A., M V Narayana (2020) "Accuracy Analysis Of An Ultrasonic Sensor Over An Open Channel Rectangular Notch For Rainwater Harvesting" international journal of scientific \& technology research volume 9, issue 01 pp 2813-2816.

[15] W. H. Strickland, R. H. King "Characteristics of ultrasonic ranging sensors in underground environment" Bureau of mines.

[16] Manabu I., Makoto S., and Shin-nosuke S. (2009) "Evaluation of Method of Measuring Distance Between Object and Walls Using Ultrasonic Sensors" Journal of Asian Electric Vehicles, Volume 7, Number 1 pp 1207-1211.

[17] Nagashima, Y., A. Ohya, and S. Yuta (1994) "Ultrasonic sensor for mobile robot to measure the normal direction of walls" The Journal of the Acoustical Society of Japan, Vol. 51, No. 1, pp 39-42. 